\section{La termodinámica: una herramienta para el análisis en química de alimentos}

\section{Thermodynamics: A tool for analysis in food chemistry}

\author{
Juan Carlos Gómez-Daza \\ Universidad del Valle
}

\section{Resumen}

La termodinámica es una ciencia básica que formula las reglas para la conversión de la materia y la energía de una forma a otra. Ella impone los límites físicos para el desarrollo y la evolución en el mundo. Los parámetros termodinámicos son importantes en el procesamiento y estabilidad del alimento. La similaridad termodinámica de los alimentos se fundamenta en diversos factores. El primero, es la función de almacenamiento fisiológico común de los más importantes componentes de las materias primas alimentarias. Segundo, es el carácter general de las interacciones intermoleculares no-específicas de las macromoléculas alimentarias. Tercero, es el mimetismo de los biopolímeros que se fundamenta en las propiedades físico-químicas muy similares de las especies de biopolímeros. La incompatibilidad termodinámica es típica en macromoléculas alimentarias. Luego, el enfoque termodinámico es altamente promisorio para el modelamiento y análisis de alimentos. Además, el equilibrio de fases juega un papel muy importante en la tecnología de alimentos. En la presente contribución, se analiza el punto de vista termodinámico en la química de alimentos. Así, el enfoque termodinámico, es central en este análisis. Se encontró que los carbohidratos y las proteínas son las moléculas más estudiadas, seguidas por el agua. En Europa, en particular Suiza, es donde más se realiza investigación en el área y donde hay contribuciones individuales destacadas. El interés por investigar en termodinámica aplicada a los alimentos, es creciente.

Palabras clave: Alimentos, análisis, equilibrio de fase, termodinámica, química.

\begin{abstract}
Thermodynamics is a basic science which states rules for conversion of matter and energy from one form to another. Thermodynamics impose physical limits for development and evolution in the world. Thermodynamic standards are important in food and stability. Thermodynamic similarity of food is founded on various factors. First, it is the function of common physiological storing of the most important components of food raw materials. Secondly, it is the general character of non-specific intermolecular interactions of food macromolecules. Third, it is the mimicry of biopolymers founded on the very similar physic-chemical properties of biopolymers species. Thermodynamic incompatibility is typical in food macromolecules. Then, the thermodynamic approach is highly promising for food modeling and analysis. In addition, phase balance plays an important role in food technology. This article analyzes the thermodynamic point of view in food chemistry. Thus, the thermodynamic approach is central in this analysis. It was found that carbohydrates and proteins are the most studied molecules, followed by water. In Europe, Switzerland in particular, is where more research is performed in the area, and where there are outstanding individual contributions. Interest in researching on thermodynamics applied to food, is growing.
\end{abstract}

Key words: Thermodynamics, analysis, chemistry, food, phase balance.
Recibido: 02/02/2016

Revisado: $15 / 02 / 2016$

Aceptado: 01/12/2016

Correspondencia de autor: juan.gomez.d@correounivalle. edu.co

(C) 2016 Universidad La Gran Colombia. Este es un artículo de acceso abierto, distribuido bajo los términos de la licencia Creative Commons Attribution License, que permite el uso ilimitado, distribución y reproducción en cualquier medio, siempre que el autor original y la fuente se acrediten.

Cómo citar:

Gómez-Daza, J.C.(2016)

La termodinámica: una herramienta para el análisis en química de alimentos. UGCiencia 22, 173-192.

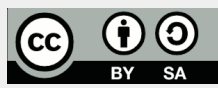




\section{Introducción}

Los alimentos son sistemas complejos, dinámicos con distribuciones no uniformes de pequeñas moléculas y biopolímeros. Aparte de sus complicaciones de heterogeneidad, compartimentación, y numerosos componentes, tienen además la naturaleza compleja de las múltiples interacciones moleculares (no idealidad) y procesos de no equilibrio (Baianu, 1992). Una descripción apropiada de un sistema químico debe considerar tres aspectos: energética, estructural y dinámico. De estos, el energético provee la contribución fundamental (Franks, 1991).

En el área de la ingeniería de alimentos, es necesario tener claridad respecto a qué son y cómo interaccionan los alimentos. Para este propósito, se busca reconocer las interacciones entre componentes de las matrices alimentarias para entender los cambios que sufren en forma natural o debido al procesamiento y al almacenamiento, para diseñar alternativas que garanticen productos de mejor calidad final (Acosta, 2008). Así mismo, en un texto clásico en la materia, se menciona que en la ciencia de los alimentos aparecen cuatro preguntas típicas: ¿Qué? - relativaalaidentidaddelos componentes en el sistema. ¿Cuánto? - la cantidad de los componentes identificados en el sistema. ¿Dónde? - en orden de localizar el componente dado dentro del sistema o en el proceso. Y por último, ¿Qué función? - para caracterizar roles y funciones fisiológicas, físicas, químicas o fisicoquímicas del componente (Pomeranz, 1985). Habría que añadir las preguntas ¿Cómo? y ¿Por qué? - que darían cabida a investigación teórica y experimental justamente para explicar las interacciones, que es donde encaja la termodinámica.

Para el análisis de las interacciones se deben considerar las variables presión $(p)$, volumen $(V)$, temperatura $(T)$, las tres variables involucradas en los cambios de fase, y por supuesto la estructura. Para analizar la estabilidad, se deben considerar las interacciones. En el análisis de la conservación de alimentos, las tres variables principales son T, pH y $a_{w}$. Se deben considerar los componentes cosmótropos y caótropos, la energética de las interacciones intermoleculares, mapa dinámico, modelo de la micela estructurada, la teoría de WLF [Williams-Landel-Ferry], entre otros (Acosta, 2008).

Todo lo anterior, permite inferir que en el área de los alimentos en general, está de forma implícita la termodinámica. Un artículo del profesor Theodore Labuza (1980) relativo a la utilidad de la termodinámica para el análisis en la química de alimentos, motiva la exploración en el tema considerando que en el título de este, aparecen dos términos claves de la teoría en consideración.

Se suele considerar el año 1824 como inicio formal de la termodinámica, cuando el joven francés Nicolás Léonard Sadi Carnot publica el trabajo clásico acerca de la potencia motriz del fuego, aunque ya en 1693, Gottfried Wilhelm Leibnitz había planteado el principio de conservación de la energía mecánica. Para llegar a su madurez, han contribuido grandes personajes entre los que podemos mencionar a Rudolf Julius Emmanuel Clausius quien establece formalmente las dos primeras leyes de la termodinámica en 1850 , y luego en 1901, Hermann Walther Nernst enuncia la tercera ley. Lo anterior en lo que respecta a la termodinámica clásica y sin desconocer a James Clerk Maxwell, Ludwig Edward Boltzmann, Hermann Ludwig Ferdinan von Helmholtz quien generalizó el principio de conservación de la energía o primera ley de la termodinámica (Gómez, 2000).

La visión del mundo está determinada en gran medida por el lenguaje que se utiliza para describirlo. La termodinámica al colocar los límites para todo lo que existe y aspire a 
existir, o los cambios que son o no posibles, se hace indispensable para dar cuenta de los cambios o transformaciones que se verifican en la naturaleza, no en vano Einstein después de meditar, concluyó que estaba convencido de que la única teoría física de contenido universal que, dentro del marco de aplicación de sus conceptos básicos, nunca será derrocada, es la termodinámica (Levenspiel, 1997).

Sin embargo, para el análisis en química de alimentos, se debe considerar que el equilibrio de fases establecido inicialmente por Josiah Willard Gibbs y llevado a la práctica por Gilbert Newton Lewis (Prausnitz, Lichtenthaler y Gomes, 2000), es una de las partes que encuentra aplicación en este artículo. Se ha hecho un trabajo interesante al respecto (Bruin, 1999). Sin embargo, es necesario aclarar que siendo los alimentos sistemas dinámicos en no equilibrio, en los casos más puristas se requiere teoría de la termodinámica de los procesos irreversibles desarrollada por el profesor Ilya Prigogine.

Siendo el agua y los carbohidratos -en especial el almidón- los constituyentes principales en la mayoría de los alimentos, no es ilógico que se hayan realizado compilaciones fundamentales en torno a estos (Rockland y Stewart, 1981; Levine y Slade, 1991; Whistler, Bemiller y Paschall, 1984). En los dos primeros trabajos sobresalen, Cornelius van den Berg y Marcus Karel entre muchos otros investigadores.

Quizá el hecho de más apoyo para el desarrollo del tema, es la identificación de trabajos sobresalientes en la misma línea de pensamiento. Así, en el intento por comprender la vida misma, se puede revisar la evolución cósmica de la entropía (la flecha del tiempo) y el origen gravitacional de la energía libre requerida para la vida, que apunta a que se debe cambiar de paradigma de "nosotros comemos alimentos" a "los alimentos se producen para comer" (Lineweaver y Egan, 2008). De igual intención pero en un nivel más particular, al analizar algunas consideraciones termodinámicas en la formulación de alimentos, cabe resaltar la similaridad termodinámica de los alimentos como una característica fundamental de los sistemas de procesos de alimentos, que determina la alta eficiencia de las tecnologías de alimentos empíricamente desarrolladas y la baja sensibilidad de las propiedades mecánicas y estructurales desde el quimo a la composición de dietas (Tolstoguzov, 2003).

También se han revisado dos conceptos termodinámicos fundamentales para la química de alimentos. Uno, son las teorías y modelos que se usan para describir y explicar los cambios físicos en el rango de temperatura $\left(T_{g}\right)$ de la transición vidrio-líquido (GLT) y el otro, es la actividad de agua. Para el primero, se describen los cambios en las propiedades mecánicas abajo y arriba de $T_{g}$, y el concepto de fragilidad, para hacer más eficiente en la formulación y control de procesos para alimentos (Champion, Le Meste y Simatos, 2000). Para el segundo concepto, se debe considerar el agua, o más propiamente su estado, expresado como $a_{w}$, un componente de los alimentos que afecta las propiedades de ingeniería de los alimentos (Lewicki, 2004).

Por otro lado, aunque se suele reconocer el agua como el más efectivo plastificante en matrices alimentarias, decreciendo la temperatura de transición vítrea y la resistencia mecánica y determinando un efecto de ablandamiento con el incremento de su concentración, se puede tener un efecto opuesto en algunos alimentos y en un rango específico de contenido de humedad o $a_{w}$, que se puede referir como efecto anti-plastificante (Pittia y Sacchetti, 2008).

Otro aspecto que merece atención son las interacciones de los biopolímeros. Investigando las propiedades de vitrificación de mezclas de biopolímeros con azúcares, se pudo demostrar que el volumen libre sigue una relación recíproca con el peso molecular promedio del biopolímero, el cual se puede relacionar con 
las asociaciones intermoleculares imperfectas que rodean las terminaciones de las moléculas. Modificando la ecuación de WLF con un término de humedad se puede describir el comportamiento de $T_{g}$, que tiene aplicación práctica en la construcción de diagramas de estado para materiales alimentarios (Kasapis, 2001). Así mismo, en otro artículo de revisión, donde se analiza las interacciones físicas débiles no específicas de los biopolímeros alimenticios (proteínas, polisacáridos) con los principales ingredientes de bajo peso molecular (azúcares, sales, surfactantes) que son claves en las propiedades funcionales de los biopolímeros como su capacidad para formar estructura, se destaca la aproximación termodinámica, basada en la combinación de métodos termodinámicos, como central en el análisis (Semenova, 2007).

Finalmente, para resaltar lo universal de la termodinámica y sus aplicaciones, se debe mencionar que se ha hecho investigación en aplicación combinada de fluidos supercríticos y tecnología de extrusión en mejoramiento de procesos de la industria de alimentos (Rizvi, Mulvaney y Sokhey, 1995; Garti, 1997), análisis de estabilidad de sistemas dispersos como las emulsiones (Dickinson, 2003), diseño de alimentos líquido-cristalinos vía simulación computacional teórica para obtener diagramas de estado (Mezzenga, Lee y Fredrickson, 2006) y aplicación de dispositivos fluídicos en ingeniería de alimentos. Todos estos trabajos tienen implícito los límites que impone la termodinámica para que su existencia sea válida.

En este trabajo, siguiendo los protocolos de búsqueda avanzada en las bases de datos ScienceDirect y EBSCO, mediante las palabras clave Thermodynamic AND Foods restringidas a abstract, título y palabras clave, se van a revisar los aspectos relacionados con la termodinámica en la química de alimentos. Se presenta una visión global de esta ciencia en la vida y luego se mostrarán trabajos realizados sobre cada uno de los temas: agua, carbohidratos complejos, proteínas, lípidos y algunos componentes funcionales. Además, se describirán algunas aplicaciones adicionales de la termodinámica en la química de alimentos. Finalmente, se discutirán los hallazgos más representativos de la revisión con miras a identificar grupos o investigadores particulares en el área e inferir tendencias.

\section{Materiales y métodos}

\section{Termodinámica, un panorama general}

La química de alimentos está íntimamente relacionada con la vida, y como la naturaleza le delegó a la segunda ley de la termodinámica el imponer los límites respecto a lo que es posible o no en el universo, necesariamente se debe hablar de ésta. De acuerdo a Lineweaver y Egan (2008): Cuando un físico iconoclástico se mueve del equilibrio y piensa generalmente acerca de la cuestión “¿Qué es la vida?”, los conceptos de entropía y energía libre juegan un rol central. Estos dos conceptos están en el corazón mismo de la termodinámica. La entropía es una propiedad derivada de la segunda ley (así como energía es derivado de la primera ley), y la energía libre es una función termodinámica ligada al concepto de equilibrio de fases y ambos conceptos pueden ser usados como criterios de espontaneidad.

Sí se visualiza la idea cualitativa de la producción de entropía en forma de pirámide para la producción de energía libre, la energía libre disponible en un nivel proviene del nivel inferior. Los niveles inferiores son prerrequisito para los niveles superiores. Los dos niveles superiores serían como formas de vida en la pirámide de producción primaria. La forma de pirámide representa una disminución de la energía libre disponible en los niveles tróficos superiores. Pasando de lo cualitativo a lo cuantitativo, incluso se podría predecir la muerte térmica del universo (Lineweaver y Egan, 2008). 
Al acotar el alcance de la termodinámica, miremos como es útil la entropía. La entropía es la noción central en la segunda ley de la termodinámica. Así, la dirección en la cual un evento puede suceder viene determinada por un aumento en la entropía. Si se consideran algunos procesos que involucran una máquina térmica y un refrigerador, dos dispositivos distintivos de la revolución industrial, los procesos se presentarían en una cadena en la cual la máquina térmica-refrigerador acoplados están conectados.

La entropía se produce en la máquina térmica. Puesto que la evolución está en continuo aumento y todo está interconectado, se puede concluir que la segunda ley de termodinámica (a través de la entropía) guía a la raza humana y su futuro (Norde, 1997). Desde un punto de vista ecológico, se puede usar la entropía para medir la perturbación del mundo, que se visualiza como un sistema críticamente auto-organizado, donde se da una relación entre el tamaño y la abundancia de cada subsistema que compone el mundo (Choi, Mazumder y Hansell, 1999). Se puede definir una termodinámica ecológica usando simulación discreta (Tollner y Kazanci, 2007). Otro punto para reconocer la importancia de la entropía, lo aporta un sistema viviente calorimétrico y termodinámico tal como los aspectos energéticos y evolucionarios de la velocidad de producción de entropía de la masa, lo así llamado enlace de disipación o función $\psi_{\mathrm{u}}$ (Lamprecht, 2003). El uso de la producción de entropía provee una perspectiva de la sensibilidad del clima y conduce a una mejor caracterización de éste $\mathrm{y}$, es un hecho más inteligente de medida global (Kleidon, 2006; Meysman y Bruers, 2007).

A nivel industrial, el método de administración de la energía en los procesos representa una responsabilidad que el químico de alimentos no puede ignorar. En un esquema típico de producción, se refleja claramente el primer principio de la termodinámica, expresado en la conocida regla de integración de procesos "más entrada-más salida". Se puede usar el método de análisis de integración de procesos en la industria de alimentos. Éste, tiene dos enfoques, hacia arriba y hacia abajo, cada uno con ventajas y desventajas (Muller, Marechal, Wolewinski y Roux, 2007).

Considerando las características de los dos enfoques, se concluye que el método descendente está desarrollado para la industria de alimentos pues no es costoso energéticamente. Sin embargo, en algunas unidades de proceso, se presentan diferencias entre los requerimientos termodinámicos de la unidad y su implementación tecnológica, por tanto el método ascendente es un complemento en el análisis (Muller, Marechal, Wolewinski y Roux, 2007). Más aún, la biomasa se puede mirar como una fuente de energía (Baratieri, Baggio, Fiori y Grigiante, 2008), lo que aumenta las posibilidades para la industria de alimentos, y esto también es termodinámica en acción.

Cuando se va a una escala siguiente en tamaño, se llega al alimento como tal. En los sistemas alimentarios, la naturaleza equilibrio/ no-equilibrio está directamente relacionada a la escala de longitud típica de la estructura del alimento y a la energía libre total del sistema. Para lograr estructuras en equilibrio verdadero, se requiere tres condiciones: (i) escalas de longitud pequeñas favoreciendo el reacomodo local sin necesidad de grandes desplazamientos, (ii) alta energía libre como fuerza motriz para la segregación, lo que minimiza las fluctuaciones térmicas y generar cambios morfológicos que minimicen la energía libre total, (iii) tiempos de relajación cortos, lo que permite obtener configuraciones de equilibrio dentro de escalas de tiempo observables (Mezzenga, 2007).

Si ahora se trata de analizar el mundo microscópico, la termodinámica también cuenta. Un balance termodinámico definido mediante el modelo Unifac modificado por Larsen, el 
modelo Pitzer-Debye-Hückel y con ecuaciones de solvatación para encontrar los coeficientes de actividad (Lebert, Dussap y Lebert, 2005), como parte de la teoría de los equilibrios de fase (Prausnitz, Lichtenthaler y Gomes, 2000).

\section{Agua y termodinámica}

Los parámetros termodinámicos y cinéticos son importantes en el procesamiento y estabilidad de los alimentos. Los parámetros termodinámicos se representan por la actividad de agua y en general se aplican a materiales de contenido de humedad media y alta, donde las moléculas pueden difundirse libremente sin restricciones. En contraste, las propiedades cinéticas se aplican principalmente en alimentos de contenido de humedad baja e intermedia, donde la movilidad molecular de los reactantes está más o menos restringida y donde el agua actúa como un plastificante de los compuestos amorfos (Anese, Shtylla, Torreggiani y Maltini, 1996). La actividad de agua tiene efecto en las cinéticas de reacción de deterioro de alimentos (Labuza, 1980a).

Para entender por qué ciertos productos son más estables que otros a un mismo valor de $a_{w}$, requiere que se elucide la estructura del agua. De particular importancia son las interacciones (hidrofílicas, hidrofóbicas) entre el agua y los componentes del alimento y el efecto de las moléculas solubles del alimento en los puentes de hidrógeno del agua solvente (Mathlouthi, 2001).

El concepto de actividad de agua $a_{w}$, surge de la teoría termodinámica. La actividad de agua puede ser definida como (Prausnitz, Lichtenthaler y Gomes, 2000):

$$
a_{w}(T, P, x)=\gamma_{w}(T, P, x) x_{w}=\frac{f_{w}(T, P, x)}{f_{w}^{0}\left(T, P^{0}, x^{0}\right)}
$$

Se puede asumir que trabajando bajo condiciones normales de temperatura ambiente y presión atmosférica, se presenta un comportamiento ideal y la relación de fugacidades se puede tomar como la relación de presiones parciales

$$
\frac{f_{w}}{f_{w}^{0}}=\frac{p_{w}}{p_{w}^{0}}
$$

Bajo este supuesto, la ecuación (1) se puede escribir como

$$
a_{w}=\frac{p_{w}}{p_{w}^{0}}=\frac{R H}{100}
$$

Donde $a_{w}$ es la actividad de agua, $x_{w}$ es la fracción molar del agua, $\gamma_{w}$ es el coeficiente de actividad para el agua, $f_{w}$ es la fugacidad del agua, $p_{w}$ es la presión de vapor del agua y $R H$ es el porcentaje de humedad relativa de la capa de aire en equilibrio con la muestra. Los supraíndice ${ }^{o}$ se refiere a las condiciones de referencia. El coeficiente de actividad se puede calcular directamente de la energía de Gibbs en exceso molar parcial del agua

$$
g_{w}^{E}=R T \ln \gamma_{w}
$$

Y para la energía de Gibbs en exceso molar total

$$
g^{E}=R T \sum_{i} x_{i} \ln \gamma_{i}
$$

Algunas aplicaciones prácticas en química de alimentos son: La deshidratación osmótica tiene un uso en la preparación de alimentos de humedad intermedia (IMF) y los así llamados alimentos de cuarta generación o mínimamente procesados. Existen grupos metodológicos usados para predecir la actividad de agua y modelos empíricos y semi-empíricos que proveen excelentes resultados y tienen amplio uso en la industria de alimentos, particularmente en el manejo de soluciones osmóticas (Sereno, Hubinger, Comesaña y Correa, 2001).

Esta área de aplicación es tan valiosa para la industria de alimentos, particularmente usando azúcar (sacarosa) debido a su disponibilidad y su fácil purificación. Una evaluación del efecto de la temperatura en el coeficiente de actividad de agua y en funciones termodinámicas de exceso 
selectas para soluciones acuosas de sacarosa, permitió encontrar una nueva ecuación de actividad de agua con numerosas aplicaciones en la tecnología de alimentos y en la industria del azúcar. Específicamente es una expresión tipo Margules pero con cuatro parámetros que resultó superior que las clásicas Uniquac y Unifac (Peres y Macedo, 1999), para predecir la actividad de agua (Starzak y Mathlouthi, 2006).

Para las soluciones acuosas de azúcar, se han realizado cálculos de coeficientes de actividad de agua basados en el número de hidratación. Para esta tarea se tiene una hipótesis implícita y es la de la contracción del volumen cuando las moléculas de agua establecen puentes de hidrógeno (Gharsallaoui, Rogé, Génotelle y Mathlouthi, 2008). También, se ha realizado un análisis termodinámico del efecto de la actividad de agua en nuez de macadamia, donde se determina la zona de mínima entropía integral, en una actividad de agua en la cual la macadamia tiene su máxima estabilidad ante la oxidación lipídica y cambios en color y textura (Domínguez, Azuara, Vernon-Carter y Beristain, 2007).

Una extensión a las aplicaciones, se puede hacer respecto a los diagramas de estado $\mathrm{y}$ la caracterización, calidad, procesamiento y estabilidad. Los cambios en el estado físico de los materiales alimentarios son a menudo fenómenos de no-equilibrio y están fuertemente relacionados al contenido de agua y la temperatura. Si se determinan valores de $T_{g}$ como función del contenido de agua y actividad de agua, se establecen diagramas de estado. Los diagramas de estado se usan para predecir el estado físico de un material alimentario a varias condiciones, y muestran la relación entre composición y temperatura que son necesarios para conseguir cambios deseados en el procesamiento o para mantener la calidad del alimento en procesamiento y almacenaje (Roos, 1995, 2003; Rahman, 2006).

\section{Carbohidratos, lípidos, proteínas, biopolímeros, otras moléculas y termodinámica}

Si bien el agua modifica el comportamiento de los otros constituyentes en el alimento, estos también presentan características de interacción que los hace llamar la atención de los investigadores. Se decidió agrupar los tres grupos constituyentes principales de los alimentos, diferentes del agua, dado que en ellos se pueden presentar macromoléculas o biopolímeros. Así, la incompatibilidad termodinámica es uno de los fenómenos más comunes en soluciones de mezclas de biopolímeros. Hay resultados que indican que se presentan interacciones favorables termodinámicamente entre polímeros cuando la sacarosa está presente. Además, parece ser que el efecto de la sacarosa en las propiedades termodinámicas de los biopolímeros en medio acuoso difiere para biopolímeros con diferente estructura.

Es posible observar incremento tanto en hidrofobicidad como en hidrofilicidad (Antipova y Semenova, 1995). Uno de los más importantes problemas en la creación de nuevos materiales basados en biopolímeros es la baja compatibilidad de estos materiales. Con base en los mismos criterios básicos de la energía libre de Gibbs, se propone que para mejorar la compatibilidad de los polímeros es adecuado producir bloques de copolímeros y la interpenetración de los circuitos de polímeros (Rogovina, 1998).

Si se revisan algunas moléculas particulares se puede establecer que, el conocimiento del comportamiento de compuestos de sabor en los sistemas multifásicos complejos (matrices alimentarias) con respecto a su estructura. Los mecanismos cinéticos y termodinámicos tienen influencia en la liberación de sabor dentro de las emulsiones y hacía la fase gaseosa en cada paso de la preparación y consumo de un producto alimentario (Landy, 
Rogacheva, Lorient y Voilley, 1998). La estabilidad de los olores en los alimentos está determinada por enlaces no covalentes de los compuestos formadores de aroma con las proteínas y los polisacáridos, siendo materia de la química supramolecular, que se apoya en la termodinámica (Golovnya et al, 2001). Las ciclodextrinas, moléculas cíclicas producidas transglucidación enzimática y ciclización del almidón, con amplia investigación, dependen de los parámetros termodinámicos como la fuerza impulsora para su complejación (Bernard, García-Mora, Díaz y Mendoza, 1999). Así mismo, el efecto de las maltodextrinas en la actividad superficial de mezclas de legumina con pequeñas moléculas surfactantes está gobernado por las interacciones competitivas con las pequeñas moléculas de surfactante en relación a la proteína y a un subsecuente cambio en las propiedades termodinámicas de ambos biopolímeros, lo cual favorece la formación de complejos ternarios (Myasoedova, Semenova, Belyakova y Antipova, 2001).

El almidón es el carbohidrato de mayor importancia en la química de alimentos y de ahí que se hayan realizado estudios avanzados para tratar de explicar su comportamiento. Así, se tomaron almidones con estructura jerárquica de gránulo con diferente contenido de amilosa para discutir la relación entre estructura y propiedades termodinámicas. Mediante diferentes aproximaciones físicas y químicas para determinar los parámetros de fusión termodinámica acoplados con métodos para investigar la estructura, proveen una razonable y exhaustiva información acerca de la organización estructural de los gránulos nativos a diferente nivel molecular y supramolecular (Bocharnikova et al, 2003).

En alimentos a base de almidón, el fenómeno de difusión del agua en estos, se ha trabajado tanto desde el punto de vista de modelos que siguen la ley de Fick tipo multicapa multifásico, así como modelos no-Fickianos llamado Modelo de demanda de Agua, en el cual se considera una presión resistiva que detiene el ingreso de agua en el cuerpo del alimento (Yahata, Fukuoka, Mihori yWatanabe, 2006; Watanabe, Yahata, Fukuoka, Sakiyama y Mihori, 2007). Este párrafo en apariencia de transferencia de masa está cobijado por una de las ecuaciones fundamentales de la termodinámica (Prausnitz, Lichtenthaler y Gomes, 2000):

$d g=\left(\frac{\partial g}{\partial P}\right)_{T, n} d P+\left(\frac{\partial g}{\partial T}\right)_{P, n} d T+\sum_{i}\left(\frac{\partial g}{\partial n_{i}}\right)_{P, T, n_{j}} d n_{i}$

La cual indica que siempre que exista un gradiente de temperatura habrá transferencia de calor, cuando exista gradiente de presión se presentará flujo de fluidos y si se presenta un gradiente en el potencial químico se verifica transferencia de masa y/o reacción química. El razonamiento anterior también aplica cuando se consideran las propiedades de formación de estructura de las emulsiones (Dalgleish, 2006) y en el efecto de separación de fases dirigido por la incompatibilidad termodinámica entre polisacáridos y las proteínas del gluten cuando se elabora pan (Fessas, Signorelli, Pagani, Mariotti, Iametti y Schiraldi, 2008). Para darle soporte a lo anterior, y al considerar que las interacciones de biopolímeros son de importancia directa para las propiedades macroscópicas (flujo, estabilidad, textura) de los productos alimentarios y donde se pueden dar casos de separación de fases gobernados por la parte entálpica -sistema gelatina-dextrano- o conducidos por efecto entrópico - interacciones de agotamiento o volumen excluido las interacciones se pueden caracterizar termodinámicamente y se puede usar para optimizar formulaciones ( De Kruif y Tuinier, 2001).

El trabajo individual más significativo en lo que tiene que ver con los aspectos termodinámicos en los alimentos, al considerar los alimentos como sistemas dispersos, y analizar los aspectos termodinámicos de la relación propiedad-composición en alimentos formulados, la implicación explícita fue 
extrapolar la anterior relación en algunos problemas de procesamiento de alimentos y se demostró la importancia de la jerarquía estructural en la formulación de sistemas dispersos alimentarios (Tolstoguzov, 2000). El siguiente paso, con una envergadura magnificada es la revisión de los aspectos termodinámicos de la funcionalidad de los biopolímeros en sistemas biológicos, alimentos y bebidas (Tolstoguzov, 2002). En este trabajo se propone el mimetismo molecular y la simbiosis molecular como los principales factores de control de la actividad termodinámica y del comportamiento de fase de los compuestos macromoleculares en los alimentos, bebidas, y el quimo. El mimetismo molecular implica un reensamble químico de las superficies hidrofílicas de las proteínas globulares con su información química oculta en el interior hidrofóbico y un bajo volumen excluido de los glóbulos.

El mimetismo contribuye a la eficiencia de las enzimas. La simbiosis molecular indica que las interacciones (atracción o repulsión) entre moléculas de biopolímeros que difieren grandemente en conformación (globular y lineal) favorece la eficiencia biológica de al menos uno de ellos. La incompatibilidad termodinámica, típica de las macromoléculas de los alimentos, cuya desnaturalización, asociación, complejación, y modificación química reduce el mimetismo y la co-solubilidad (Tolstoguzov, 2002).

Al considerar termodinámicamente la funcionalidad del almidón en los alimentos, se revisa la paradoja en formulación de alimentos. ¿Por qué son las pastas de almidón inestables?. $\mathrm{Y}$, por el contrario, ¿Por qué son los alimentos a base de almidón de calidad reproducible? ¿Por qué es la sinergia y el antagonismo típicos en las mezclas de polisacáridos?. Y desde luego, también existe una explicación termodinámica a esto. Así, se concluye que las interacciones no-covalentes, no-específicas de las macromoléculas están sujetas a la inmensa diversidad estructural y a la similaridad termodinámica de los alimentos (Tolstoguzov, 2003a, 2004).

Generalizando lo anterior a la función de los polisacáridos, se considera el rol funcional de los polisacáridos en el surgimiento de la vida y la evolución de sus funciones usando las propiedades termodinámicas de biopolímeros nativos y desnaturalizados modernos, donde se llega a la hipótesis general que se puede formular como "todo lo que un animal pueda hacer, las moléculas de biopolímeros pueden hacerlo" (Tolstoguzov, 2003b). Le siguen dos trabajos con un grado más de ambición intelectual, en el primero plantea un escenario hipotético para el origen de la vida como una evolución solidaria de proto-biopolímeros en un ambiente de polisacáridos. Este se basa en las propiedades termodinámicas de biopolímeros modernos, sus mezclas y conjugados. Se incluyen las siguientes etapas arbitrarias en el surgimiento de la vida: (i) el mundo de polisacáridos; (ii) el mundo de conjugados conteniendo mezclas heterogéneas química y estructuralmente de proto-biopolímeros; y (iii) el mundo de proto-células (Tolstoguzov, 2004). Si el primero justificó el por qué fueron, en el segundo se trata de justificar el por qué son. Entonces, a lo anterior le suma que la tendencia conductora esencial de la vida es mantener la homeostasis, esto es, sobrevivir, y que los polisacáridos son los responsables del contacto ecológico de las entidades vivientes, esto es, para la nutrición y la inmunidad universal no específica, de ahí que los polisacáridos continúen siendo los polímeros más abundantes del planeta (Tolstoguzov, 2004a).

Para los lípidos también hay termodinámica. Así, las transformaciones moleculares y las propiedades cinéticas de las grasas, que pueden ser representadas como triacilgliceroles (TAGS), cuya molécula particular es un triéster del glicerol y tres ácidos grasos (Sato, Ueno y Yano, 1999). Quizá lo más representativo del tema son el polimorfismo y las transformaciones 
polimórficas, que están incluidas en el análisis de la cristalización de las grasas. Todo el análisis se fundamenta en la función termodinámica energía libre de Gibbs

$$
G=h-T s
$$

Los procesos involucrados en el análisis de la cristalización y almacenamiento, las relaciones energéticas para formas polimórficas y la descripción de las barreras de energía en las transformaciones de las grasas, están bien documentados (Himavan, Starov y Stapley, 2006).

Para las proteínas también se encuentra trabajo interesante. Tolstoguzov tiene su hipótesis para el origen de la estructura globular de éstas. Explica que la incompatibilidad termodinámica de los biopolímeros que difieren en estructura y composición química puede trazar la tendencia evolutiva hacia composición de aminoácidos preferible proveyendo macromoléculas más compactas con superficies más similares químicamente (Tolstoguzov, 1999), y se ha mostrado un mecanismo para proteína de suero (Reiffers-Magnani, Cuq y Watzke, 2000).

Para tener una visión general de la intervención de la termodinámica en la explicación química del comportamiento de las proteínas, se presentó un diagrama de transferencia de energía libre de proteína desde el agua. La transferencia de energía libre de las proteínas hacia soluciones cosolventes provee una descripción cuantitativa de la influencia del cosolvente en las transiciones entre los diferentes estados de la proteína, esto es, plegada o sin pliegue o agregada o no agregada. Se tiene un modelo para predecir la exclusión estérica y la contribución de interacción diferencial (Mc Clements, 2001).

En las proteínas, fundamentado siempre en la termodinámica (barreras energéticas, incompatibilidad termodinámica, separación de fases), también se ha trabajado en varios contextos. Así, en el análisis de películas donde se forman capas adsorbidas desde mezclas de proteínas (Damodaran, 2004), en el estudio termodinámico y de comportamiento de partición en proteínas por agregación térmica de metil celulosa en solución acuosa (Spelzini, Rigatusso, Farruggia y Picó, 2005), estudio termodinámico de las reacciones metabólicas en una solución de proteína acuosa (Kamiyama, Toshima, Matsushita y Kimura, 2005), en la preparación de emulsiones múltiples basadas en la incompatibilidad termodinámica de proteína de suero desnaturalizada por calor y soluciones de pectina (Kim, Decker y McClements, 2006). Para el estudio del salting in (disolución) y el salting out (precipitación) en las proteínas a partir de las interacciones entre macromoléculas y iones y su efecto en la estabilidad basado en las series de Hofmeister implican termodinámica (Baldwin, 1996; Zhang y Cremer, 2006), las interacciones segregativas entre gelatina $\mathrm{y}$ proteína de suero polimerizada (Fitzsimons, Mulvihill y Morris, 2008).

\section{Resultados}

\section{Termodinámica y otras aplicaciones}

En este apartado, se presentan de manera genérica las aplicaciones más representativas de la termodinámica en temas que tienen relación implícita con la química de alimentos. Así:

Efecto del procesamiento en el valor nutritivo y la termodinámica del alimento. Se consideran frutas y vegetales, cereales y granos, carnes/ alimentos de mar, productos lácteos, grasas y aceites (Prochaska, Nguyen, Donat y Piekutowski, 2000).

Simulación numérica de los procesos termodinámicos y de dinámica de fluidos durante el tratamiento con alta presión de sistemas alimentarios fluidos. Se utilizan técnicas de dinámica de fluidos computacional (CFD) en el análisis de tratamiento con alta presión de alimentos, útil en escalamiento (trazado y diseño de dispositivos). Se encontró 
que si la parte térmica es heterogénea, afecta el proceso (Hartmann, 2002).

Incremento de tamaño de una palomita de maíz. Se usa la termodinámica para analizar cómo se produce este popular pasaboca. Se trata el mecanismo como una expansión adiabática y se obtiene predicción cualitativa correcta (Quinn Sr, Hong y Both, 2005).

Efectos de la nanoescala en las tecnologías de producción de alimentos. En la Tabla 1 se muestran efectos de energía. Se formulan mecanismos e hipótesis y se encuentra que aparentemente, los procesos combinados abren nuevas posibilidades en el control de los cambios estructurales en el producto y permiten reducir el consumo de potencia de producción y organizan nuevas nano-tecnologías de producción de alimentos (Burdo, 2005).

Determinación de los parámetros termodinámicos de la formación de complejos entre malvidin-3-O-glucósido y polifenoles. Efectos de copigmentación en vinos rojos. Se encontró que el proceso de copigmentación tiene carácter exotérmico. El cambio de entalpía es el mismo en cualquier paso de la complejación. Sin embargo, la reducción del término entrópico es mayor a mayor estequiometria, la energía libre de Gibbs cambia y, así, la estabilidad del complejo decrece ligeramente con el incremento en la estequiometria (Kunsági-Mate, Szabo, Nikfardjam y Kollár, 2006).

Ingeniería de procesos en alimentos avanzada para modelar procesos y alimentos reales: La metodología "Safes" (aproximación sistemática a sistemas de ingeniería de alimentos). Se propone un nuevo modelo para desarrollar procesos y alimentos donde es posible incorporar información acerca de todos los aspectos (termodinámico, estructural, químico y bioquímico, y también mecanístico). Reconoce la complejidad del alimento y permite coordinar la información acerca de la estructura, composición, calidad, termodinámica, etc. En herramientas adecuadas para el desarrollo. Implica manejo termodinámico matricial (Fito, LeMaguer, Betoret y Fito, 2007).

Solución calorimétrica: Una nueva perspectiva en el proceso de disolución de alimentos en polvo. Se investiga la utilidad de la entalpía de disolución para estudiar el comportamiento termodinámico de alimentos en polvo solubles. Se sugiere que la disolución es un fenómeno complejo y que la nueva técnica puede conducir a un mejor entendimiento y optimización del proceso (Marabi et al, 2007).

Tabla 1. Efectos energéticos de las nanotecnologías (Burdo, 2005)

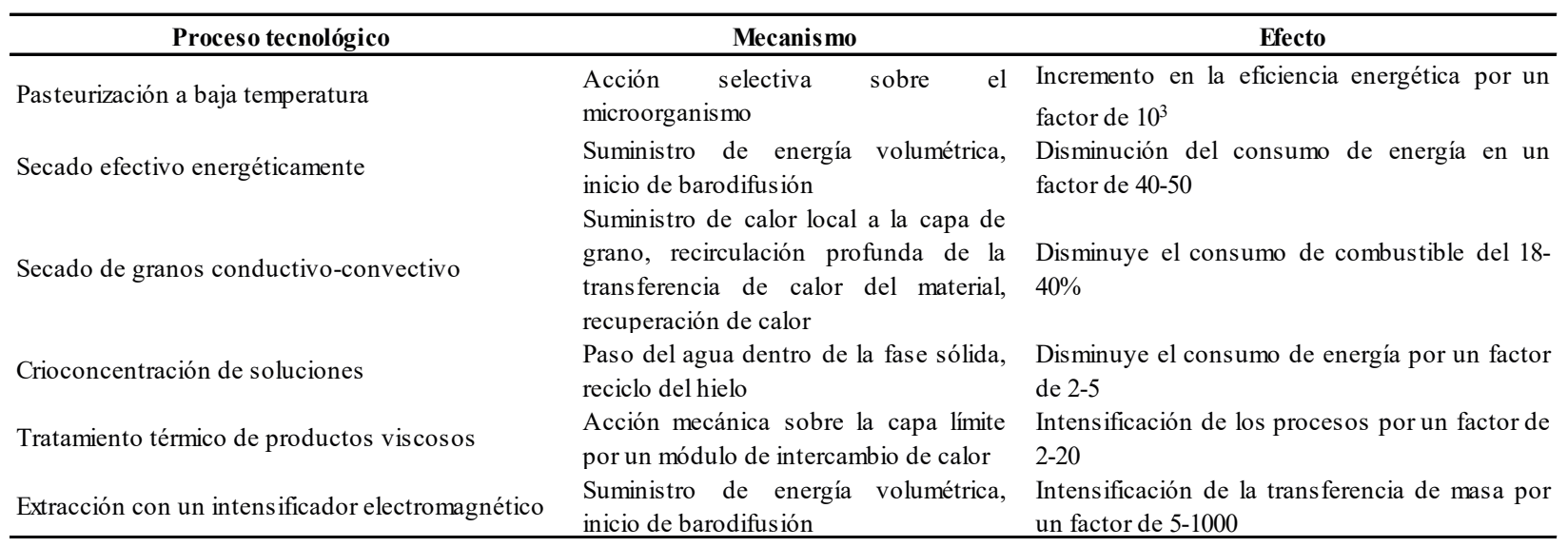


Límites termodinámicos para la operación de enfriamiento profundo en túnel de platos. Se usan dos indicadores para el desempeño del sistema: la potencia exergética y la destrucción de exergía específica. Se usa un modelo endoreversible, se considera no sólo el flujo de masa del alimento sino también el nivel de temperatura del alimento a la salida (Ducoulombier, Sorin y Teyssedou, 2007).

Recientes avances en el uso de alta presión (HP) como una técnica de procesamiento efectiva en la industria de alimentos. Las ventajas clave de las aplicaciones de HP a los sistemas alimentarios son la independencia del tamaño y la geometría de la muestra durante el procesamiento, posible por el tratamiento a baja temperatura y la disponibilidad de una tecnología amigable ambientalmente libre de desechos (Norton y Sun, 2008). Tiene muy en cuenta el estado físico del agua a las diferentes condiciones.

Aditivos alimentarios obtenidos por extracción supercrítica de fuentes naturales. Se modela con ecuación de contribución de grupos la termodinámica compleja (Espinosa, Diaz y Brignole, 2008).

\section{Discusión de resultados}

Para poder inferir tendencias e identificar grupos, se presentan las figuras 1, 2, 3 y 4 .

Figura 1. Distribución del número de artículos por tema considerado

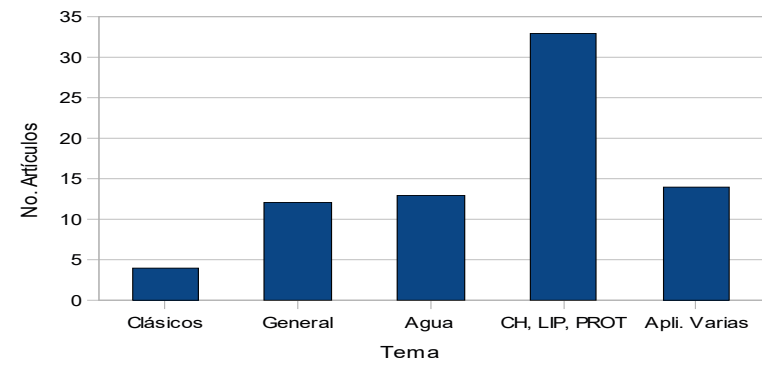

(Fuente: el autor)
Figura 2. Distribución del número de artículos por año

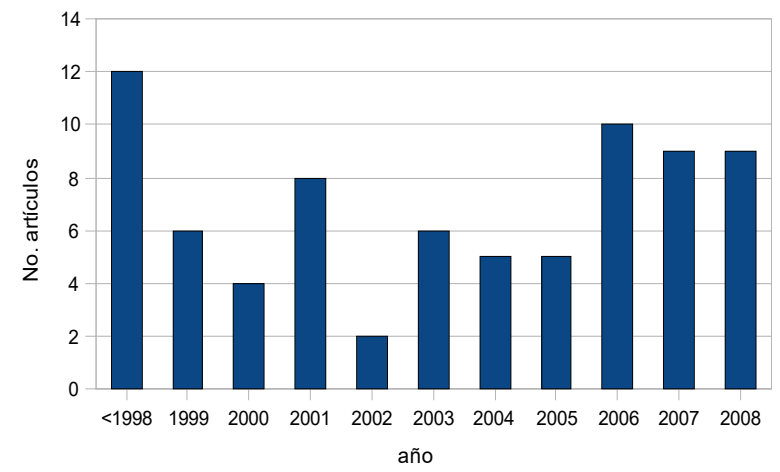

(Fuente: el autor)

Figura 3. Distribución del número de artículos por país

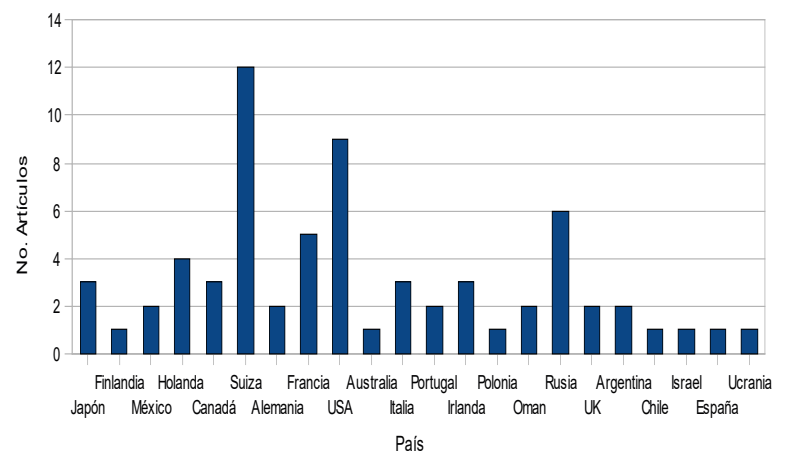

(Fuente: el autor)

Figura 4. Distribución del número de artículos para cada componente por periodo de tiempo

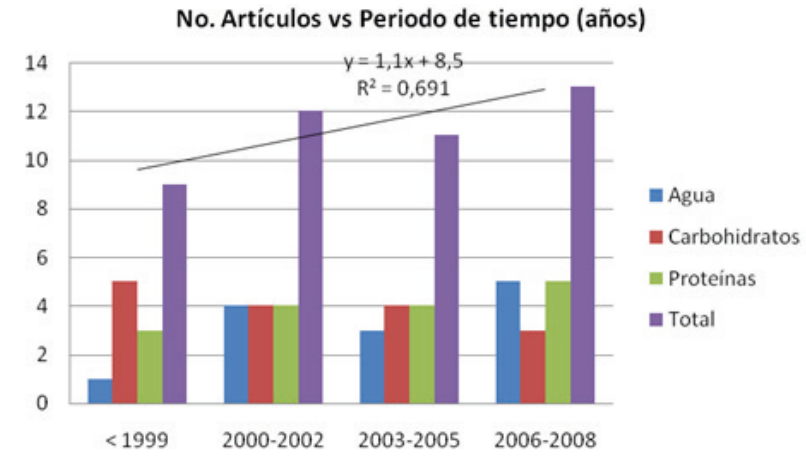

(Fuente: el autor) 
De la figura 1 se puede inferir que la mayor productividad en investigación termodinámica asociada a la química de alimentos está en el tema carbohidratos, lípidos y proteína, quizá por el mismo hecho de estar agrupadas.

De la figura 2 se identifica el año 2006 como el de mayor interés en el área por parte de los investigadores y de acuerdo al número de artículos publicados.

La figura 3 indica que los países más destacados en la investigación de la aplicación de la termodinámica en la química de alimentos son en orden decreciente, Suiza $>$ USA $>$ Rusia.

Finalmente, de la figura 4 se puede inferir que hay una tendencia no bien definida (con base en el $\mathrm{R}^{2}$ ) en el análisis termodinámico de los temas de la química de alimentos.

A continuación, se presenta de forma más detallada la distribución del número de artículos por tema (figura 5).

Figura 5. Distribución porcentual de los artículos por tema específico

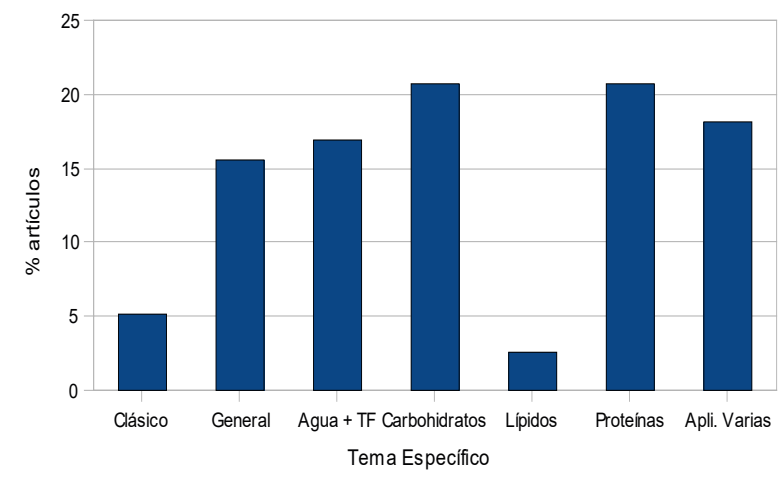

(Fuente: el autor)

De la figura 5 se puede inferir que los carbohidratos y las proteínas tienen el mismo nivel de interés por parte de los investigadores en el tema.
La baja representación de los lípidos quizá es debida a que como grupo químico particular presenta la mayor dificultad para su análisis termodinámico. Se podría considerar las publicaciones acerca de emulsiones dentro de este grupo para compensar la desproporción, pero de forma explícita es lo que se encontró.

A continuación se presenta la distribución por región de la literatura consultada (figura 6).

Figura 6. Distribución porcentual de la literatura por región

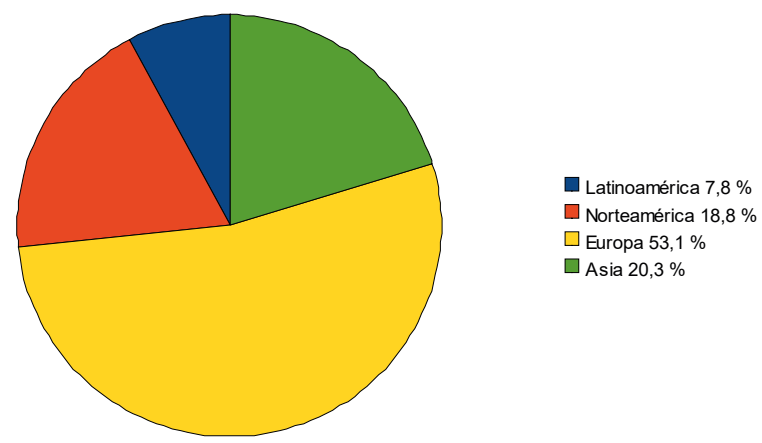

(Fuente: el autor)

La figura 6 indica que Europa es la región donde la termodinámica en la química de alimentos despierta el mayor interés, quizá por el enfoque académico que trabajan las escuelas tanto en las universidades o quizá por la formación particular de los autores.

A continuación se presenta la identificación (no exhaustiva) de los investigadores más representativos en varios temas de interés respecto a la aplicación de la termodinámica en la química de alimentos (tabla 2). 
Tabla 2. Identificación de temas e investigadores en el área (fuente: el autor)

\begin{tabular}{|c|c|c|}
\hline Autor & Ubicación & Tema de interés \\
\hline Tolstoguzov, V. & Nestlé - Suiza & $\begin{array}{l}\text { Aspectos termodinámicos, } \\
\text { funcionalidad } \mathrm{CH} \text { (almidón) }\end{array}$ \\
\hline Lewicki, P. & U. Warsau - Polonia & $\begin{array}{l}\text { Propiedades de ingeniería de los } \\
\text { alimentos }\end{array}$ \\
\hline Kasapis, S. & U. Qaboos - Oman & Teoría WLF \\
\hline Semenova, M.G. & Academia Ciencias - Rusia & Biopolímeros \\
\hline Dickinson, E. & U. Leeds - UK & Sistemas dispersos \\
\hline Mathlouthi, M. & U. Reims - Francia & Actividad de agua \\
\hline Pittia, P. & U. teramo - Italia & Efecto antiplastificante del agua \\
\hline Mezzenga, R. & U. Fribourg - Nestlé & $\begin{array}{l}\text { Sistemas complejos, equilibrio y } \\
\text { no-equilibrio }\end{array}$ \\
\hline Lamprecht, I. & U. Berlín - Alemania & Sistemas vivientes \\
\hline Bruin, S. & Unilever - Holanda & Equilibrio de fases \\
\hline Rogovina, L. & $\begin{array}{l}\text { Inst. Organoelementos - } \\
\text { Rusia }\end{array}$ & $\begin{array}{l}\text { Sistemas multicomponentes, } \\
\text { biopolímeros }\end{array}$ \\
\hline Damodoran, S. & $\begin{array}{l}\text { U. W is consin-Madison - } \\
\text { USA }\end{array}$ & Proteínas \\
\hline McClements, D.J. & U. Massachusetts - USA & Proteínas \\
\hline Roos, Y. & U. Hels inki - Finlandia & Diagramas de estado \\
\hline Rahman, M. & $\begin{array}{l}\text { Colegio ciencias marinas - } \\
\text { Oman }\end{array}$ & Diagramas de estado \\
\hline Kruif, C.G. & $\begin{array}{l}\text { Inst. Inv. Alim. - NIZO - } \\
\text { Holanda }\end{array}$ & $\begin{array}{l}\text { Interacción proteína- } \\
\text { polis acáridos }\end{array}$ \\
\hline Stapley, & U. Laughborough - UK & Lípidos \\
\hline Sato, K. & U. Hiroshima - Japón & lípidos \\
\hline Dalgleish, & U. Guelph - Canadá & Estructura emulsiones \\
\hline $\begin{array}{l}\text { Champion, Le } \\
\text { Meste, Simatos }\end{array}$ & ENSBANA - Francia & Transiciones vítreas \\
\hline Labuza, T. & U. Minnesota - USA & Actividad de agua \\
\hline Norton y Sun & U. Dublín - Irlanda & $\begin{array}{l}\text { Alta presión en industria de } \\
\text { alimentos }\end{array}$ \\
\hline Watanabe, $\mathrm{H}$. & U. Tokio - Japón & Difusión en almidones \\
\hline Hartmann, Chr. & U. München - Alemania & Simulación numérica \\
\hline Cremer, P.S. & U. Texas A \& M - USA & Series Hofmeister \\
\hline Baldwin, R.L. & U. Stanford - USA & Series Hofmeister \\
\hline Burdo, O. & Acad. Odessa - Ucrania & $\begin{array}{l}\text { Nanotecnología en la industria } \\
\text { alimentos }\end{array}$ \\
\hline Brignole, E.A. & U. Nal. Del Sur - Argentina & $\begin{array}{l}\text { Fluidos supercríticos en ing. } \\
\text { Alimentos }\end{array}$ \\
\hline Aguilera, J.M. & U. Pont. Católica - Chile & $\begin{array}{l}\text { Dispositivos microfluídicos en } \\
\text { industria alimentos }\end{array}$ \\
\hline Fito, $\mathrm{P}$. & U. Polit. Valencia - España & Metodología SAFES \\
\hline
\end{tabular}




\section{Conclusiones}

La termodinámica como ciencia de carácter universal ha permeado las áreas de estudio de la química de alimentos. Los carbohidratos y las proteínas tienen un interés equivalente para los investigadores en el área. Los lípidos representan un nicho de investigación potencial para el análisis termodinámico dada su bajo nivel de publicaciones.

Hay un hecho curioso implícito en la revisión realizada y es, que varios de los mejores trabajos en el área lo han realizados investigadores de manera individual, aparte que hay un buen componente de investigación privada (Nestlé, Unilever) y además femenina, casos María Semenova, Rafaela Mezzenga, Paola Pittia.

Con base en el número de publicaciones y los alcances de éstas, se puede identificar a Vladimir Tolstoguzov en Suiza (cuna de los Bernoulli) como el gurú de los 90s e inicios del siglo XXI. Nestlé en Suiza, al parecer invierte una cantidad importante en la investigación del análisis termodinámico en la química de alimentos, así tiene en su nómina a investigadores de la talla de Tolstoguzov y Mezzenga.

A grandes rasgos, se pueden identificar los países con un tema específico, así: Francia (Mathlouthi) con la actividad de agua; Suiza (Tolstoguzov, Mezzenga) con los carbohidratos; Rusia (Semenova, Rogovine) con los biopolímeros; UK (Himawan, Starov y Stapley) con los lípidos; USA (Damodoran, McClements) con las proteínas.

La termodinámica en la química de alimentos es un área que está dentro de los intereses de los investigadores en cualquiera de sus temas y es significativa principalmente para los países europeos. El $53 \%$ de la investigación se ha realizado en Europa. Norteamérica y Asia están al mismo nivel en intensidad de investigación acerca del tema.

\section{Referencias}

Acosta, H.A. (2008). Notas del curso Química de Alimentos Avanzada. Cali: Universidad del Valle.

Anese, M., Shtylla, I., Torreggiani, D. y Maltini, E. (1996). Water activity and viscosity-relations with glass transition temperaturas in model food systems. Thermochimica Acta, 275, 131-137.

Antipova, A.S. y Semenova, M.G. (1995). Effect of sucrose on the thermodynamic incompatibility of different biopolymers. Carbohydrate Polymers, 28, 359-365.

Baianu, I.C. (1992). Physical Chemistry of Food Processes. Volume 1. Westport, USA: AVI Publishing.

Baldwin, R.L. (1996). How Hofmeister ion interactions affect protein stability. Biophysical Journal, 71, 2056-2063.

Baratieri, M, Baggio, P, Fiori, L. y Grigiante, M. (2008). Biomass as an energy source: Thermodynamic constraints on the perfomance of the conversión process. Bioresource Technology, 99, 7063-7073.

Bernad, M.J, Gracia-Mora, J, Díaz, D. y Mendoza, G. (1999). Molecular interactions and thermodynamic aspect of the complexation reaction between Gentian Violet and several cyclodextrins. Journal of Inclusion Phenomena and Macrocyclic Chemistry, 34, 1-18.

Bocharnikova, I, Wasserman, L.A., Krivandin, A.V., Fornal, J., Blaszczak, W., Chernykh, V.Y., Schiraldi, A. y Yuryev, V.P. (2003). Structure and thermodynamic melting parameters of wheat starches with different amylose content. Journal of Thermal Analysis and Calorimetry, 74, 681-695. 
Bruin, S. (1999). Phase equilibria for food product and process design. Fluid Phase Equilibria, 158-160, 657-671.

Burdo, O.G. (2005). Nanoscale effects in food-production technologies. Journal of Engineering Physics and Thermophysics, 78(1), 90-96.

Champion, D, Le Meste, M. y Simatos, D. (2000). Review Towards an improved understanding of glass transition and relaxations in foods: molecular mobility in the glass transition range. Trends in Food Science \& Technology, 11, 41-55.

Choi, J.S, Mazumder, A. y Hansell, R. I. C. (1999). Measuring perturbation in a complicated, thermodynamic world. Ecological Modelling, 117, 143-158.

Dalgleish, D.G. (2006). Keynote paper Food emulsions-their structures and structure-forming properties. Food Hydrocolloids, 20, 415-422.

Damodaran, S. (2004). Adsorbed layers formed from mixtures of proteins. Current Opinion in Colloid \& Interface Science, 9, 328-339.

De Kruif, C.G. y Tuinier, R. (2001). Polysaccharide protein interactions. Food Hydrocolloids, 15, 555-563.

Dickinson, E. (2003). Review Hydrocolloids at interfaces and the influence an the properties of dispersed systems. Food Hydrocolloids, 17, 25-39.

Domínguez, I.L, Azuara, E., Vernon-Carter, E.J. y Beristain, C.I. (2007). Thermodynamic analysis of the effect of wáter activity on the stability of macadamia nut. Journal of Food Engineering, 81, 566-571.
Ducoulombier, M, Sorin, M. y Teyssedou, A. (2007). Thermodynamic bounds for food deep chilling tray tunnel operation. International Journal of Thermal Science, 46, 172-179.

Espinosa, S, Diaz, M.S. y Brignole, E.A. (2008). Food additives obtained by supercritical extraction from natural sources. The Journal of Supercritical Fluids, 45, 213-219.

Fessas, D, Signorelli, M, Pagani, A, Mariotti, M, Iametti, S. y Schiraldi, A. (2008). Guidelines for buckwheat enriched bread: Thermal analysis approach. Journal of Thermal Analysis and Calorimetry, 91, 9-16.

Fito, P, LeMaguer, M, Betoret, N. y Fito, P.J. (2007). Advanced food process engineering to model real foods and processes: The "SAFES" methodology. Journal of Food Engineering, 83, 173-185.

Fitzsimons, S.M, Mulvihill, D.M. y Morris, E.R. (2008). Segregative interactions between gelatin and polymerised whey protein. Food Hydrocolloids, 22, 485-491.

Franks, F. (1991). New perceptions of wáter relationships in foods. In: Levine, H. y Slade, L. (1991). Water relationships in foods: Advances in the 1980s and trends for the 1990s. New York: Plenum Press.

Garti, N. (1997). Revieww Progress in stabilization and transport phenomena of doublé emulsions in food applications. Lebesm.-Wiss. U.-Technol., 30, 222-235. 
Gharsallaoui, A, Rogé, B., Génotelle, J. y Mathlouthi, M. (2008). Relationships between hydration number, wáter activity and density of aqueous sugar solutions. Food Chemistry, 106, 1443-1453.

Golovnya, R.V, Yuryev, V.P., Kalistratova, E.N, Wasserman, L.A., Terenina, M.B., Krikunova, N.I. y Misharina, T.A. (2001). Thermodynamic characteristics of supramolecular complexes of aroma compounds with ordered structures of polysaccharides of corn starch and its cryotextures. Russian Chemical Bulletin, International Edition, 50(2), 300-303.

Gómez, J. (2000). Apuntes históricos-Apéndice Módulo Termodinámica, Especialización en ingeniería de procesos de alimentos y biomateriales UNAD_CAMPINAS. Bogotá: Corcas editores.

Hartmann, Chr. (2002). Numerical simulation of thermodynamic and fluid-dynamic processes during the high-pressure treatment of fluid food systems. Innovative Food Science \& Emerging Technologies, 3, 11-18.

Himawan, C, Starov, V.M. y Stapley, A.G.F. (2006). Thermodynamic and kinetic aspect of fat crystallization. Advances in Colloid and Interface Science, 122, 3-33.

Kamiyama, T, Toshima, Y, Matsushita, T. y Kimura, T. (2005). Thermodynamic study of metabolic reactions in an aqueous protein solution. Journal of Thermal Analysis and Calorimetry, 82, 207-212.
Kasapis, S. (2001). Advanced topics in the application of the WLF/free volumen theory to high sugar/biopolymer mixture: a review. Food Hydrocolloids, $15,631-641$.

Kim, H, Decker, E.A. y McClements, D.J. (2006). Preparation of multiple emulsions base don thermodynamic incompatibility of heat-denatured whey protein and pectin solutions. Food Hydrocolloids, 20, 586-595.

Kleidon, A. (2006). The climate sensitivity to human appropriation of vegetation productivity and its thermodynamic characterization. Global and Planetary Change, 54, 109-127.

Kunsági-Máte, S, Szabo, K., Nikfardjam, M.P. y Kollár, L. (2006). Determination of the thermodynamic parameters of the complex formation between malvidin-3-O-glucoside and polyphenols. Copigmentation effect in red wines. Journal of Biochemical and Biophysical Methods, 69, 113-119.

Labuza, T.P. (1980). Enthalpy / Entropy compensation in food reactions. Food Technology, 34(2), 67-77.

Labuza, T.P. (1980a). The effect of wáter activity on reaction kinetics of food deterioration. Food Technology, 34(4), 36-41.

Lamprecht, I. (2003). Calorimetry and thermodynamics of living systems. Thermochimica Acta, 405, 1-13.

Landy, P, Rogacheva, S, Lorient, D. y Voilley, A. (1998). Thermodynamic and kinetic aspects of transport of small molecules in dispersed systems. Colloids and Surfaces B: Biointerfaces, 12, 57-65. 
Lebert, I, Dussap, C.G. y Lebert, A. (2005). Combined physico-chemical and wáter transfer modelling to predict bacterial growth during food processes. International Journal of Food Microbiology, 102, 305-322.

Levenspiel, O. (1997). Fundamentos de termodinámica. México: Prentice-Hall Hispanoamericana.

Levine, H. y Slade, L. Editors. (1991). Water relationships in foods: Advances in the 1980s and trends for the 1990s. New York: Plenum Press.

Lewicki, P.P. (2004). Water as the determinant of food engineering properties. A review. Journal of Food Engineering, 61, 483-495.

Lineweaver, C.H. y Egan, C.A. (2008). Review: Life, gravity and the second law of thermodynamics. Physics of Life Reviews, 5, 225-242.

Marabi, A, Mayor, G, Raemy, A, Bauwens, I, Claude, J, Burbidge, A.S. Wallach, R. y saguy, I.S. (2007). Solution calorimetry: A novel perspective into the dissolution process of food powders. Food Research International, 40, 1286-1298.

Mathlouthi, M. (2001). Water content, wáter activity, wáter structure and the stability of foodtuffs. Food Control, 12, 409-417.

McClements, D.J. (2001). Estimation of steric exclusión and differential interaction contributions to protein transfer free energies in aqueous cosolvent solutions. Food Hydrocolloids, 15, 355-363.
Meysman, F.J.R. y Bruers, S. (2007). A thermodynamic perspective on food webs: Quantifying entropy production within detrital-based ecosystems. Journal of Theorical Biology, 249, 124-139.

Mezzenga, R, Lee, W.B. y Fredrickson, G.H. (2006). Review Design of liquid-crystalline foods via field theoretic computer simulations. Trends in Food Science \& Technology, 17, 220-226.

Mezzenga, R. (2007). Equilibrium and non-equilibrium structures in complex food systems. Food Hydrocolloids, 21, 674-682.

Muller, D.C.A, Marechal, F.M.A., Wolewinski, T. y Roux, P.J. (2007). An energy management method for the food industry. Applied Thermal Engineering, 27, 2677-2686.

Myasoedova, M.S, Semenova, M.G., Belyakova, L.E. y Antipova, A.S. (2001). Surface activity at the planar interface in relation to the thermodynamics of intermolecular interactions in the ternary system: maltodextrin-small-molecule surfactant-legumin. Colloids and Surfaces B: Biointerfaces, 21, 179-189.

Norde, W. (1997). Energy and entropy: a thermodinamic approach to sustainability. The Environmentalist, 17, 57-62.

Norton, T. y Sun, D.-W. (2008). Recent advances in the use of high pressure as an effective processing technique in the food industry. Food Bioprocess Technol, 1, 2-34. 
Peres, A.M. y Macedo, E.A. (1999). Prediction of thermodynamic properties using a modified UNIFAC model: application to sugar industrial systems. Fluid Phase Equilibria, 158-160, 391-399.

Pittia, P. y Sacchetti, G. (2008). Antiplasticization effect of wáter in amorphous foods. A review. Food Chemistry, 106, 1417-1427.

Pomeranz, Y. (1985). Functional properties of food components. Orlando: Academic Press, Inc.

Prausnitz, J.M, Lichtenthaler, R.N. y Gomes de Acevedo, E. (2000). Termodinámica molecular de los equilibrios de fases. 3 Ed. Madrid: Prentice-Hall.

Prochaska, L.J, Nguyen, X.T, Donat, N. y Piekutowski, W.V. (2000). Effects of food processing on the thermodynamic and nutritive value of foods: literatura and database survey. Medical Hypotheses, 54(2), 254-262.

Quinn Sr, P.V, Hong, D.C. y Both, J.A. (2005). Increasing the size of a piece of popcorn. Physica A, 353, 637-648.

Rahman, M.S. (2006). State diagram of foods: Its potential use in food processing and product stability. Trends in Food Science y Technology, 17, 129-141.

Reiffers-Magnani, C.K, Cuq, J.L. y Watzke, H.J. (2000). Depletion flocculation and thermodynamic incompatibility in whey protein stabilised $\mathrm{O} / \mathrm{W}$ emulsions. Food Hydrocolloids, 14, 521-530.

Rizvi, S.S.H, Mulvaney, S.J. y Sokhey, A.S. (1995). The combined application of supercritical fluid and extrusión technology. Review. Trends in Food Sciece \& Technology, 6, 233-240.
Rockland, L.B. y Stewart, G.F. Editors. (1981). Water activity: Influences on food quality. New York: Academic Press.

Rogovina, L.Z. (1998). Approaches to multicomponent of biopolymers. Food Hydrocolloids, 12, 325-331.

Roos, Y. (1995). Characterization of food polymers using state diagrams. Journal of Food Engineering, 24, 339-360.

Roos, Y. (2003). Thermal analysis, state transitions and food quality. Journal of Thermal Analysis and Calorimetry, 71, 197-203.

Sato, K, Ueno, S. y Yano, J. (1999). Molecular interactions and kinetic properties of fats. Progress in Lipid Research, 38, 91-116.

Semenova, M.G. (2007). Thermodynamic analysis of the impact of molecular interactions on the functionality of food biopolymers in solution and in colloidal systems. Food Hydrocolloids, 21, 23-45.

Sereno, A.M, Hubinger, M.D, Comesaña, J.F. y Correa, A. (2001). Prediction of wáter activity of osmotic solutions. Journal of Food Engineering, 49, 103-114.

Spelzini, D, Rigatusso, R, Farruggia, B. y Picó, G. (2005). Thermal aggregation of methyl cellulose inaqueous solutions: a thermodynamic study and protein partitioning behaviour. Cellulose, 12, 293-304.

Starzak, M. y Mathlouthi, M. (2006). Temperature dependence of wáter activity in aqueous solutions of sucrose. Food Chemistry, 96, 346-370. 
Tollner, E.W. y Kazanci, C. (2007). Defining an ecological thermodynamics using discrete simulation aproaches. Ecological Modelling, 208, 68-79.

Tolstoguzov, V. (1999). Origins of globular structure in proteins. FEBS Letters, 444, 145-148.

Tolstoguzov, V. (2000). Foods as dispersed systems: Thermodynamic aspects of composition-property relationships in formulated food. Journal of Thermal Analysis and Calorimetry, 61, 397-409.

Tolstoguzov, V.(2002). Thermodynamic aspects of biopolymer functionality in biological systems, foods, and beverages. Critical Review in Biotechnology, 22(2), 89-174.

Tolstoguzov, V. (2003). Review: Some thermodynamic considerations in food formulation. Food Hydrocolloids, 17, $1-23$.

Tolstoguzov, V. (2003). Thermodynamic considerations on starch functionality in foods. Carbohydrate Polymers, 51, 99-111.

Tolstoguzov, V. (2003). Thermodynamic considerations on polysaccharide functions. Polysaccharides came first. Carbohydrate Polymers, 54, 371-380.

Tolstoguzov, V. (2004). Why were polysaccharides necessary?. Origins of Life and evolution of the Biosphera, 34, 571-597.

Tolstoguzov,V.(2004). Why are polysaccharides necessary?. Food Hydrocolloids, 18, 873-877.
Watanabe, H, Yahata, Y, Fukuoka, M., Sakiyama, T. y Mihori, T. (2007). The thermodynamic basis for the relative wáter demand model that describes non-Fickian wáter diffusion in starchy foods. Journal of Food Engineering, 83, 130-135.

Whistler, R.L, Bemiller, J.N. y Paschall, E.F. Editors. (1984). Starch: Chemistry and Technology. Second Edition. Orlando: Academic Press, Inc.

Yahata, Y, Fukuoka, M, Mihori, T. y Watanabe, H. (2006). Journal of Food Engineering, 76, 490-499.

Zhang, Y. y Cremer, P.S. (2006). Interactions between macromolecules and ions: the Hofmeister series. Current Opinion in Chemical Biology, 10, 658-663. 